\title{
Structural Basis of Atrial Arrhythmogenesis in Metabolic Syndrome
}

\author{
Shaleka Agrawal ${ }^{1}$, Girish Ramlugun ${ }^{1}$, Kevin Jamart ${ }^{1}$, James Kennelly ${ }^{1}$, Jesse Ashton ${ }^{2}$, Gregory \\ Sands $^{1}$, Manuel Zarzoso ${ }^{3}$, Jichao Zhao ${ }^{1}$ \\ ${ }^{1}$ Auckland Bioengineering Institute, ${ }^{2}$ Physiology Department, University of Auckland, Auckland, \\ New Zealand
}

${ }^{3}$ Department of Physiotherapy, University of Valencia, Valencia, Spain

\begin{abstract}
Individual components of metabolic syndrome (MetS) have been correlated with atrial fibrillation $(A F)$, but as a whole, the exact mechanism underlying the increased susceptibility of $A F$ still remains unclear. This study identifies key structural substrates in a robust obesogenic dietary model of MetS in the rabbits. The rabbit atria from both MetS and controls ( $N=3$ each) were processed and incubated in wheat germ agglutinin (WGA) to label cell membranes and collagen. Confocal microscopy was used to image the tissue. The collagen and cell membranes were segmented using a robust machine learning architecture, $V$-net. Quantification of fibrosis was done by calculating the ratio of total pixels of collagen to those of atrial tissue in each of the segmented images. Cell hypertrophy measurements were calculated by measuring means of individual cell diameters. We discovered atrial dilation, increased collagen, cell hypertrophy and reduction in axial-tubules in MetS atria. These are established arrhythmogenic phenotypes which might lead to increased AF susceptibility.
\end{abstract}

\section{Introduction}

Metabolic syndrome (MetS) is a fast-rising global epidemic, due to the increase in sedentary lifestyles and excess calorie intake [1]. It is defined as a cluster of cardiovascular diseases and metabolic abnormalities including obesity, hypertension, diabetes and dyslipidemia. Individual components of MetS increase the risk of new-onset of atrial fibrillation (AF), the most common sustained arrhythmia in humans, often leading to increased complications and death. The mechanism underlying the elevated risk of $\mathrm{AF}$ in MetS is not completely understood. Atrial structural and electrical remodelling are known to trigger and maintain $\mathrm{AF}$ and may hold the key to identifying the AF mechanism [2]. Atrial structural remodelling contributes to high $\mathrm{AF}$ susceptibility, with fibrosis formation being one of the most important factors [3].

High-resolution confocal imaging is a powerful tool to give a deep insight into structural remodelling by identifying fibrosis using wheat germ agglutinin (WGA) [4], followed by image segmentation and quantification of fibrosis. However, segmentation is challenging due to high variability and complexity of structural features and image artifacts. The conventional approach is labour-intensive and highly sensitive to image quality, hence we used a robust automated segmentation method. We propose a convolutional neural network $(\mathrm{CNN})$ for segmenting the cell membranes and quantifying fibrosis in MetS and Control atria [5].

\section{Methods}

The study conformed to the EU directive 2010/63 on the protection of animals used for scientific purposes, and was approved by the Institutional Animal Care and Use Committee of the Universitat de València (2015/VSC/PEA/00049). The animal model was developed and euthanized in Universitat de València. Adult New Zealand white rabbits were fed ad libitum with a high-fat, high-sucrose diet $(85 \%$ standard diet, $10 \%$ hydrogenated coconut oil and 5\% pork lard) to induce MetS (N=3), alongside age-matched Controls $(\mathrm{N}=3)$ which were fed with standard chow [6].

\subsection{Tissue preparation and Staining}

Male New Zealand White rabbit's left and right atrial (LA and RA) free walls were fixed in $4 \%$ paraformaldehyde, sunk in $30 \%$ sucrose solution, snap-frozen in liquid nitrogen using methanol, and stored at $-80^{\circ} \mathrm{C}$. The tissue was defrosted, then embedded in optimal cutting temperature liquid OCT for cryosectioning. Thin strips $(200 \mu \mathrm{m})$ of transmural tissue were then mounted on Superfrost plus glass slides and cleared using a novel approach of advanced $C U B I C$ reagent-1. Reagent-1 is a mixture of urea (25 wt\% final concentration), $80 \mathrm{wt} \%$ Quadrol (25 wt\% final concentration), Triton X-100 (15 wt $\%$ final concentration) and $\mathrm{dH}_{2} \mathrm{O}$ [7]. After clearing, the tissue is washed in potassium buffer solution (PBS) and was incubated in WGA, followed by another wash in PBS. 


\subsection{Data acquisition}

Confocal imaging was then performed using a confocal laser scanning microscope (Nikon Eclipse TE300, with a resolution of $\left.\sim 0.2 \times 0.2 \times 0.5 \mu \mathrm{m}^{3}\right)$ equipped with 488 excitation lines. This process was performed for both MetS and control hearts $(\mathrm{N}=3$ each), for $1 \mathrm{~mm}$ section each from the free wall of LA and RA. Approximately 125 images (512x512 pixels) were acquired from each atrium of the hearts.

\subsection{Segmentation}

The acquired images were segmented for further processing, using the V-net CNN [8]. Ground truth for training the CNN was segmented using Huang method in Image J [9]. To create a bigger training dataset two image augmentation approaches were used: orthogonal rotation and flipping around the horizontal or vertical axes. These were chosen as they do not produce image artifacts.
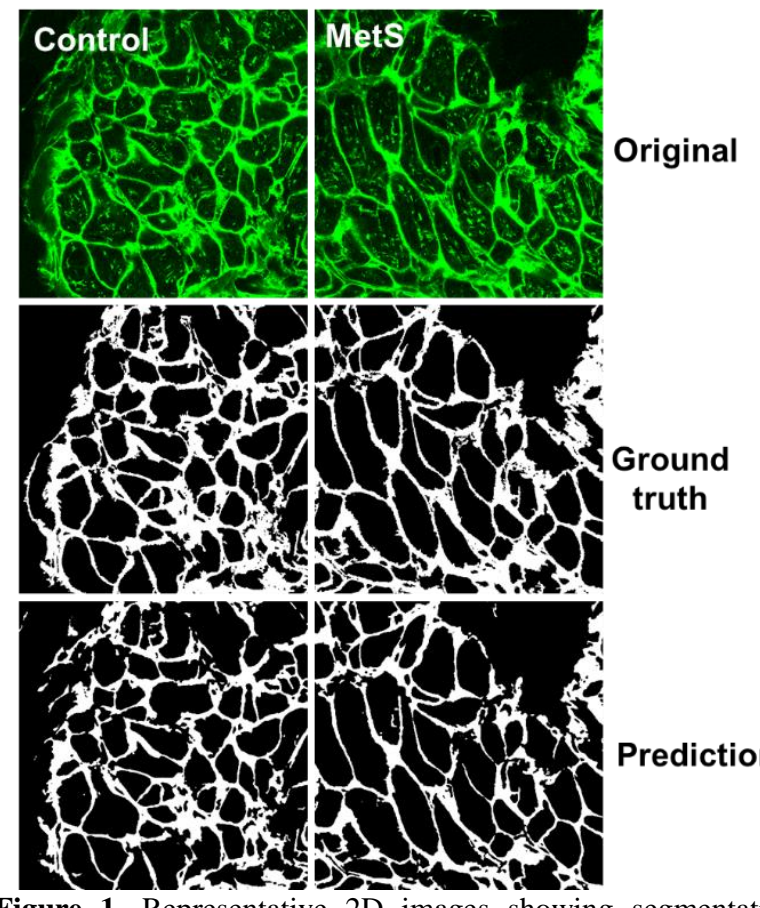

Figure 1. Representative 2D images showing segmentation results for the LA using the most widely used machine learning approach, V-net, on Control (left) and MetS images (right). Top: Original images; Middle: Ground truth used for training the network; Bottom: Preditions made by the network.

TensorFlow framework was used to implement the CNN, and for the first iteration of training the weights were initialized using variance scaling. Training was carried out using gradient descent in batches of 13 images. Weights were updated every batch and training was terminated when the Dice similarity coefficient (DSC) for the test set did not increase by at least $0.1 \%$ after 50 iterations from its current best epoch. The best model with the highest test mean DSC was saved.

The primary segmentation performance metric used for biological segmentation was the widely adopted DSC. Intersection of union (IoU), a common metric for semantic segmentation was also used. Both of these measure overlap areas of mutual class assignment but differ slightly in terms of formulation. Class scores were averaged over each image yielding DSC and IoU, the respective class scores were further averaged over the test data set resulting in the mean DSC and mean IoU. Each model produced a mean DSC score over the test data set, with the best mean DSC model being saved as the top-performance model.

The V-net segmentation approach achieved a high level of performance with the DSC converged around epoch 100 and scoring a test mean DSC of 0.8747 and IoU score of 0.7772 . Predictions were visually almost identical to the ground truth (Figure 1), we will improve the $\mathrm{CNN}$ in future by incorporating a bigger training dataset.

\subsection{Data analysis}

The segmented images were processed using a custommade Matlab code. The number of pixels were counted and divided by the total area occupied by cells in each image to get the percentage fibrosis, as shown in Figure 3A. Cell area and cell count were quantified by identifying clusters of background pixels in the range of 600 to 5000 pixels. This specific range was chosen for processing transverse cells as the expected area occupied by each cell (accounting for hypertrophy) would lie in the range 75 to $600 \mu \mathrm{m}^{2}$. Individual cell areas were averaged per image to analyse the level of cell hypertrophy (Figure 3B). Axialtubules were identified as clusters of foreground pixels lying within previously identified cell boundaries. The percentage of axial-tubules per cell was quantified (by dividing the total number of foreground pixels by the total number of pixels comprising the cell) to realise their downregulation (Figure 3C).

\section{Results}

At the organ level a significant increase in chamber volume was observed in MetS. We observed dilation in LA diameter with $11.06 \pm 0.07 \mathrm{~mm}$ in MetS compared to $9.40 \pm 0.03 \mathrm{~mm}$ in control.

At the tissue level, a two-fold increase in fibrotic tissue was observed in MetS compared to Control (Figure 3A). Intercellular space was expanded with more extracellular matrix (ECM). Extensive interstitial and patchy fibrosis (Figure 2 A \& D) was observed in comparison to control. A thick layer of fat cells was observed close to the epicardium in both MetS and control. 

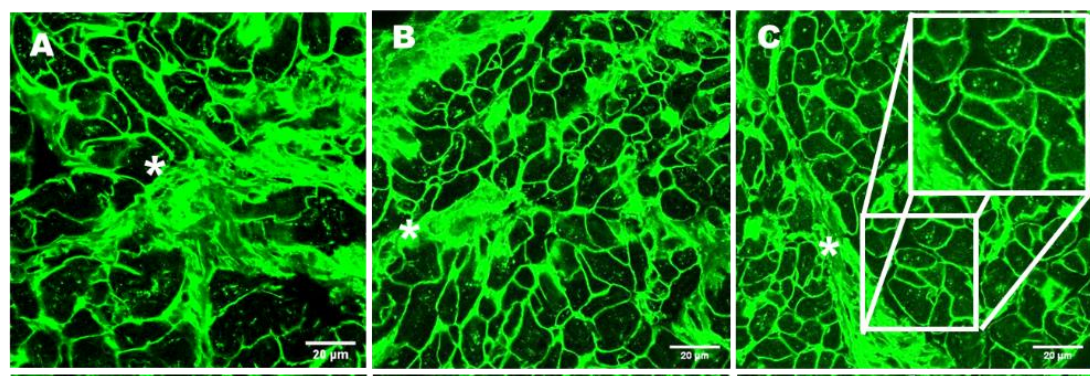

MetS
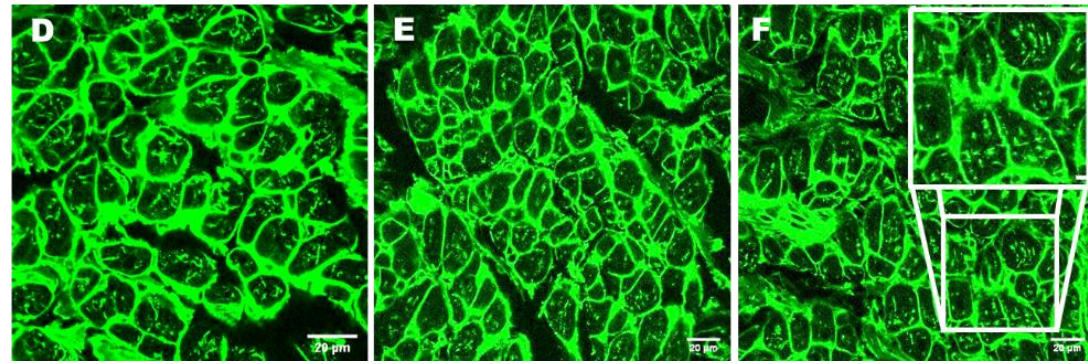

Control

Figure 2. Raw confocal images from the free wall of the left atrium of MetS and Control rabbits showing the different aspects of structural remolleding: A) Patchy and interstitial fibrosis in MetS in comparison to Control in D); Similarly B) showing cell hypertrophy in MetS against E) in Control; Finally C) is showing the downregulation of axial-tubules against Control in F), with a zoomed-in region shown in a white box; The white asterisks show patchy fibrosis.

At the cellular level, remodeling occurred with hypertrophy around the scar tissue and close to the endo and epicardium. Additionally, hypertrophy in cardiomyocytes with collagen deposition was apparent, Figure 2 B \& E. Figure 3B illustrates the average area of MetS LA cells.

At the sub-cellular level, there was a reduction in axialtubules (Figure $2 \mathrm{C} \& \mathrm{~F}$ ) in MetS by a factor of two (Figure $3 \mathrm{C}$ ). All the aforementioned changes were not consistent across both atria.

The LA of MetS had hypertrophied cells and prolific extracellular space compared to control. These inflamed cells were concentrated around fibrotic regions along with the pericardial and endocardial layers. Additionally in the RA the hypertrophy was more pronounced in MetS compared with control, with a layer of fat cells in between the pericardium and myocardium.

\section{Discussion}

This study demonstrates that concomitant diseases in MetS add to the development of an arrhythmogenic substrate in the atria, increasing AF inducibility. MetS was shown to be associated with enhanced ECM, increased cell size and reduced axial-tubules. These structural changes would display impaired regional and global atria conduction increasing AF susceptibility.

To date, single cardiac risk factors such as diabetes mellitus, obesity, hypertension, and dyslipidemia have been shown to independently lead to electrical and structural remodeling of the atria [10-13]. Clinical observations suggest that a certain combination of risk factors might lead to more pronounced arrhythmogenic atrial remodeling. The evaluations of the present study accounting for the effects of obesity, insulin resistance, dyslipidemia and hypertension appear to support this theory.

Our preliminary study gives an insight into the effect of multiple concomitant risk factors on arrhythmogenic atrial substrates. Previous studies have demonstrated in a variety of animal models [1][12-15] that AF susceptibility increased in structurally remodeled atria, alongside heterogeneities in conduction. Dilated atria would contribute to electrical remodeling by changing the dispersion of repolarization. An increase in interstitial fibrosis was consistent with changes observed in patients with AF and AF animal models [13-17]. Furthermore, the documented structural changes in the atria, especially the increase in ECM along with endomysial and replacement fibrosis formation, might lead to conduction abnormalities by disrupting side-to-side electrical conduction, thus increasing AF susceptibility. LA dilation was observed which has been reported to prolong the action potential leading to polymorphic atrial tachycardia degenerating to AF [1][14]. We observed reduced tubular junctions in the atria, previously shown to be leading to a marked spatiotemporal heterogeneity of systolic $\mathrm{Ca}^{2+}$ waves [1819]. The importance of the tubule network has been long recognized in the ventricular myocytes but it still remains controversial for atria. However, a recent study has shown transverse tubules in mammalian atrial myocytes including humans [20]. More interesting, a 50\% higher density of axial-tubule junctions than of transverse tubules were identified in intact live human atrial myocytes using superresolution microscopy imaging [21]. In this study, 


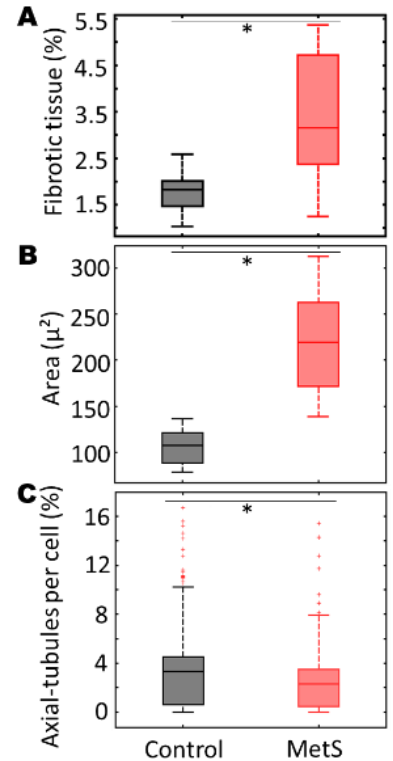

Figure 3. Results of analysis of cellular structure and properties performed on the segmented images, acquired from the free wall of the LA; where A) Percentage fibrosis in the MetS rabbits compared to controls; B) Average area of cells; C) Percentage axial-tubules per cell; where ${ }^{*} \mathrm{p}<0.001$.

more axial-tubules were observed in comparison to transverse-tubules, this will be quantified in future. This remodeling at the sub-cellular level is a maladaptive hypertrophy response occurring due to the remodeling at the cellular level.

\section{Conclusion}

MetS was found to add atrial arrhythmogenic phenotypes including cellular hypertrophy, increased interstitial and patchy fibrosis, and a reduction in axial-tubules, potentially increasing propensity for AF. Future research is warranted to determine the role of electrical remodeling on the atria of MetS and control rabbits.

\section{Acknowledgements}

This study was supported by the Health Research Council of New Zealand.

\section{References}

[1] N Milici, "A Short History of the Metabolic Syndrome Definition," Proc. Rom. Acad., vol. Series B, pp. 13-20, 2010.

[2] Schotten Ulrich, et al. "Pathophysiological Mechanisms of Atrial Fibrillation: A Translational Appraisal." Physiological Reviews 91.1 (2011): 265-325

[3] Zhao Jichao, et al. "Three-dimensional Integrated Functional, Structural, and Computational Mapping to Define the Structural "Fingerprints" of Heart-Specific Atrial Fibrillation Drivers in Human Heart Ex Vivo." Journal of the American Heart Association 6.8 (2017): e005922.

[4] Emde, B., et al. "Wheat Germ Agglutinin Staining as a Suitable
Method for Detection and Quantification of Fibrosis in Cardiac Tissue after Myocardial Infarction." European Journal of Histochemistry: EJH 58.4 (2014).

[5] Xiong Zhaohan, et al. "Fully Automatic Left Atrium Segmentation from Late Gadolinium Enhanced Magnetic Resonance Imaging using a Dual Fully Convolutional Neural Network." IEEE Transactions on Medical Imaging 38.2 (2018): 515-524.

[6] Arias-Mutis Óscar Julián, et al. "An Experimental Model of DietInduced Metabolic Syndrome in Rabbit: Methodological Considerations, Development, and Assessment." JoVE (Journal of Visualized Experiments) 134 (2018): e57117.

[7] Susaki Etsuo A., et al. "Advanced CUBIC Protocols for Whole-Brain and Whole-Body Clearing and Imaging." Nature Protocols 10.11 (2015): 1709.

[8] Milletari Fausto, et al. "V-net: Fully Convolutional Neural Networks for Volumetric Medical Image Segmentation." 2016 Fourth International Conference on 3D Vision (3DV). IEEE, 2016.

[9] Huang, Liang-Kai, and Mao-Jiun J. Wang. "Image Thresholding by Minimizing the Measures of Fuzziness." Pattern Recognition 28.1 (1995): 41-51.

[10] Lau Dennis H., et al. "Short-Term Hypertension is Associated with the Development of Atrial Fibrillation Substrate: A Study in an Ovine Hypertensive Model." Heart Rhythm 7.3 (2010): 396-404.

[11] Lau Dennis H., et al. "Hypertension and Atrial Fibrillation: Evidence of Progressive Atrial Remodeling with Electrostructural Correlate in a Conscious Chronically Instrumented Ovine Model." Heart Rhythm 7.9 (2010): 1282-1290.

[12] Mahajan Rajiv, et al. "Electrophysiological, Electroanatomical, and Structural Remodeling of the Atria as Consequences of Sustained Obesity." Journal of the American College of Cardiology 66.1 (2015): 1-11.

[13] Linz Dominik, et al. "Cathepsin A Mediates Susceptibility to Atrial Tachyarrhythmia and Impairment of Atrial Emptying Function in Zucker Diabetic Fatty Rats." Cardiovascular Research 110.3 (2016): 371-380

[14] Neuberger Hans-Ruprecht, et al. "Development of a Substrate of Atrial Fibrillation During Chronic Atrioventricular Block in the Goat." Circulation 111.1 (2005): 30-37.

[15] Li Danshi, et al. "Promotion of Atrial Fibrillation by Heart Failure in Dogs: Atrial Remodeling of a Different Sort." Circulation 100.1 (1999): 87-95.

[16] Verheule Sander, et al. "Alterations in Atrial Electrophysiology and Tissue Structure in a Canine Model of Chronic Atrial Dilatation Due to Mitral Regurgitation." Circulation 107.20 (2003): 2615-2622.

[17] Verheule Sander, et al. "Loss of Continuity in the Thin Epicardial Layer Because of Endomysial Fibrosis Increases the Complexity of Atrial Fibrillatory Conduction." Circulation: Arrhythmia and Electrophysiology 6.1 (2013): 202-211.

[18] Eisner, David, et al. "Calcium Flux Balance in the Heart." Journal of Molecular and Cellular Cardiology 58 (2013): 110-117.

[19] Lenaerts, Ilse, et al. "Ultrastructural and Functional Remodeling of the Coupling Between $\mathrm{Ca}^{2+}$ Influx and Sarcoplasmic Reticulum $\mathrm{Ca}^{2+}$ Release in Right Atrial Myocytes from Experimental Persistent Atrial Fibrillation." Circulation Research 105.9 (2009): 876-885.

[20] Richards, Mark Antony, et al. "Transverse Tubules are a Common Feature in Large Mammalian Atrial Myocytes Including Human." American Journal of Physiology-Heart and Circulatory Physiology 301.5 (2011): H1996-H2005.

[21] Brandenburg, Sören, et al. "Axial Tubule Junctions Activate Atrial $\mathrm{Ca}^{2+}$ Release Across Species." Frontiers in Physiology 9 (2018): 1227.

Address for correspondence.

Dr. Jichao Zhao

Auckland Bioengineering Institute

University of Auckland, New Zealand

E-mail: j.zhao@auckland.ac.nz 